\title{
Huge nodular goitre with retrosternal extension- a rare case report
}

\begin{abstract}
Retrosternal goitre is very rare condition which account for 5 to $20 \%$ of thyroidectomy patients. Though rare, it can be better managed surgically. In this article we report a rare case of 55year old female who had nodular goiter with huge retro sternal extension associated with hyperthyroidism. Majority of retrosternal thyroid can be removed safely by conventional cervical approach. Our patient had around $12 \mathrm{~cm}$ mass which was impossible to remove it from conventional approach and hence total thyroidectomy by a combined cervical/sternotomy approach was planned. Procedure was uneventful and patient is doing fine in 5 months of follow-up with no complications. The role of thyroidectomy in patients with retrosternal goitre provides yet another area of surgical controversy.
\end{abstract}

Keywords: retrosternal goitre, sternotomy
Volume 7 Issue 6 - 2018

\author{
Mohammad Akheel,' Rajnish Nagarkar, ${ }^{2}$ \\ Shirsendu Roy ${ }^{2}$ \\ 'Department of Surgical Oncology, CHL-CBCC Cancer Centre, \\ India \\ ${ }^{2}$ Curie Manavata cancer centre, India
}

Correspondence: Mohammad Akheel, Department of Surgical Oncology, CHL-CBCC Cancer Centre, Indore, India, Tel 08886030256,Email drakhelomfs@gmail.com

Received: April 05, 2017| Published: November 13, 2018

\section{Introduction}

Retrosternal goiter is defined when $50 \%$ of the thyroid is below the thoracic inlet. This can also be called as intra thoracic goiter. Retrosternal goiter is a very rare condition which account for 5 to $20 \%$ of thyroidectomy patients. ${ }^{1}$ Mediastinal extension is more common in huge goiters with a peak incidence in $5^{\text {th }}$ to $6^{\text {th }}$ decade. This is usually rare when the patient has associated hyperthyroidism which may increase the risk of complications. The reported incidence of goiters with thyroid malignancy is around 3 to $17 \% \cdot{ }^{1,2}$ Retrosternal goitre occurs when the thyroid enlarges downwards into the chest. Although the great majority of retrosternal goitres are extensions from the neck, pure intra thoracic goitres are very rare. Retrosternal goitres are more likely to be left sided and very rarely a left sided cervical goitre descends into the right side of the chest which is called a "crossed substernal goitre. ${ }^{2}$ With few exceptions huge goiters can be removed by cervical approach but less than $2 \%$ patients require cervical/sternotomy approach. All these factors will influence the perioperative management of the patient. In this article we report a rare case of 55year old female who had nodular goiter with huge retrosternal extension associated with hyperthyroidism. The tumor was removed surgically by a combined cervical/sternotomy approach.

\section{Case report}

A 55 year old reported to our cancer centre with a chief complaint of difficulty in breathing and eating from 2 years. General examination was insignificant. Local examination revealed a diffuse swelling of left lobe of thyroid (Figure 1). Her medical history revealed hyperthyroidism from 2years. A provisional diagnosis of Thyroid swelling was made. Radiological examination was done. Computed tomography of neck revealed $12 \times 8 \times 7 \mathrm{~cm}$ retrosternal mass arising from left lobe of thyroid with tracheal compression (Figure 2). There was no cervical lymphadenopathy. Thyroid function test (T3, T4 and TSH) were done. T3 was $2.11 \mathrm{nmol} / 1$, T4 was $228.86 \mathrm{nmol} / 1$ and TSH was less than $0.06 \mathrm{uIU} / \mathrm{Ml}$. Fine needle aspiration of the lesion gave a final diagnosis of nodular colloid goitre with cystic changes. Since T4 was high, patient was started on Tab Neomarkazole $2.5 \mathrm{mg}$, Tab Inderal $80 \mathrm{mg}$ and Lugol Iodine solution orally for 5 days to avoid Thyrotoxicosis. After 5 days T3 was $0.98 \mathrm{nmol} / \mathrm{L}$, T4 was $180.36 \mathrm{nmol} / \mathrm{L}$ and TSH was less than $0.05 \mathrm{Uiu} / \mathrm{Ml}$. Patient was taken for surgery. Fibreoptic laryngoscopy was done to visualize the tracheal patency. Awake intubation was done. General anesthesia was administered. Necklace incision was given $2 \mathrm{~cm}$ above the sternum. Strap muscles were dissected. Thyroid gland was exposed. First left thyroid gland was mobilized followed by right thyroid gland. Bilateral recurrent laryngeal nerve was identified and preserved (Figure 3). The left thyroid mass was dissected. Sternotomy was done to enucleate the retrosternal mass from anterior mediastinum. Thyroid gland along with retrosternal mass was excised (Figure 4). Homeostasis was achieved. Drains were kept and closure was done in layers. Postoperatively patient was started on Tab Neomaracazole $2.5 \mathrm{mg}$ and Tab Inderal $80 \mathrm{mg}$. Postoperative T4 was $145 \mathrm{nmol} / \mathrm{L}$. Patient was discharged on $4^{\text {th }}$ postoperative day and patient is doing fine in 5 months of followup with no complications.

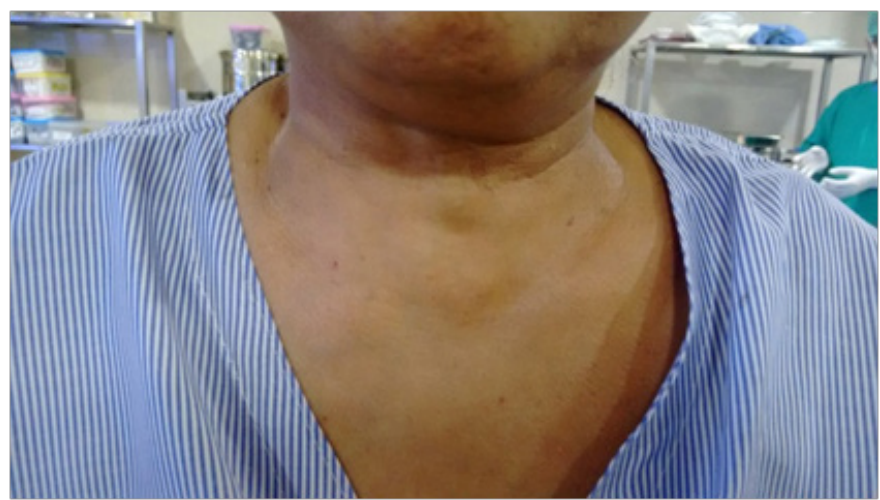

Figure I Diffuse swelling of left lobe of thyroid. 


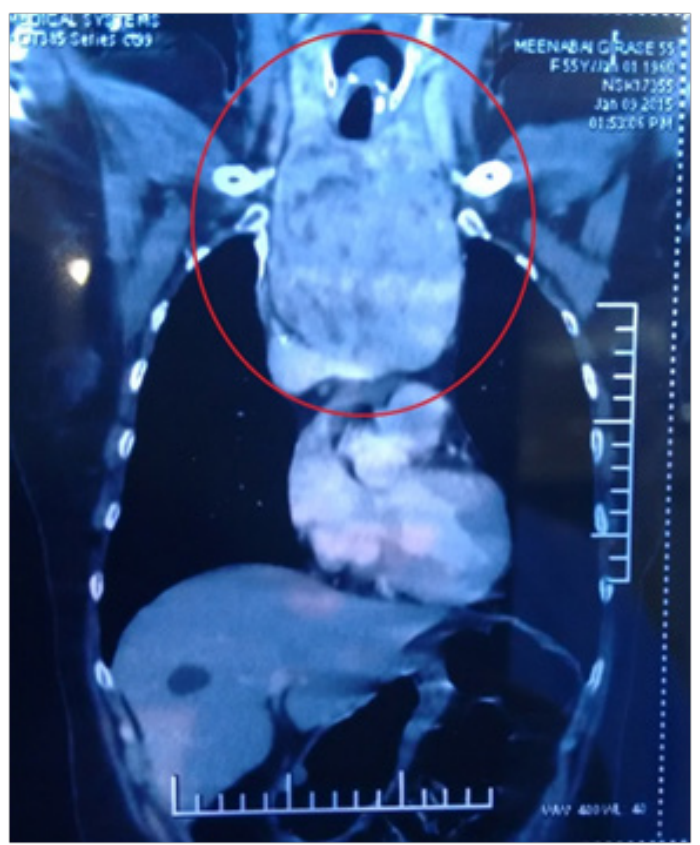

Figure 2 Computed tomography of neck revealed $12 \times 8 \times 7 \mathrm{~cm}$ retrosternal mass arising from left lobe of thyroid with tracheal compression.

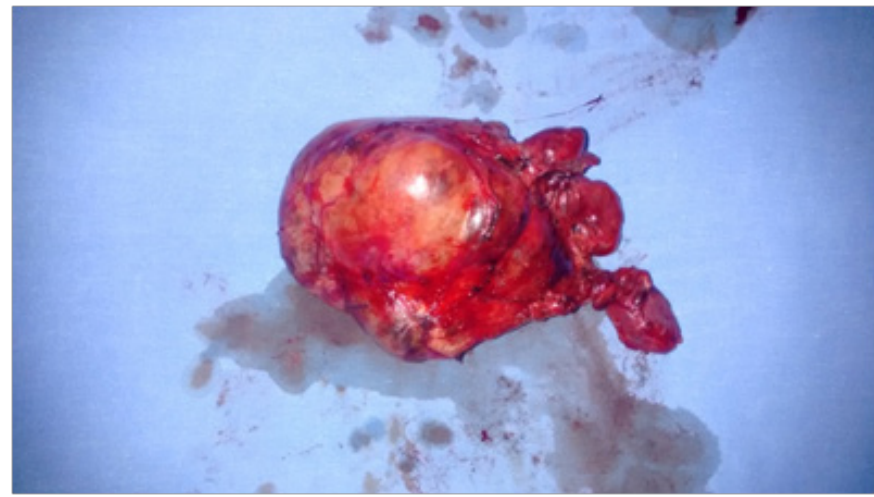

Figure 3 Excised Retrosternal goitre.

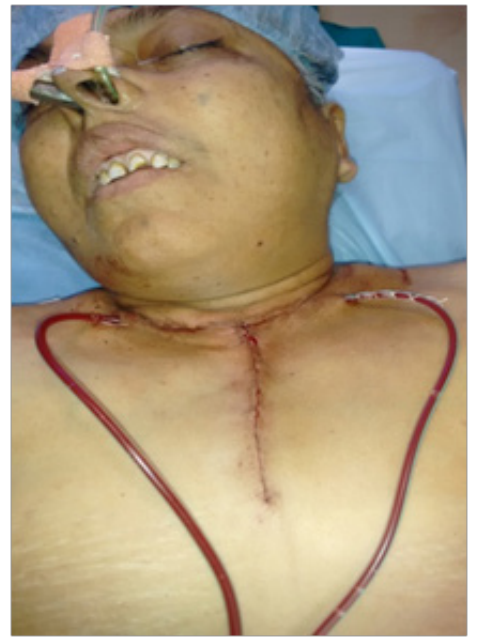

Figure 4 Closure done.

\section{Discussion}

The definition of retrosternal goiter is not uniform and often varies among authors. Goldenberg and Lindskog defined retrosternal goiter as a lesion of the thyroid gland extending downward the fourth thoracic vertebra on chest imaging or a structure with an inferior margin extending down to the level of the arch of the aorta. ${ }^{3,4}$ According to Katlic and colleagues retrosternal goiter is defined when more than $50 \%$ of the mass lies distally to the thoracic outlet. Patients often complains with slow and progressive growth commonly seen in 5th or 6 th decade of life. When the age of clinical presentation advances there is increased medical co-morbidity implying that surgery at an earlier stage may be associated with reduced complications related to coexisting disease. Other symptoms can be cough, dyspnea, dysphagia, stridor, and symptoms of choking which are absolute indications for surgery. ${ }^{5-7}$ Our patient had all these clinical symptoms. Radiological Imaging indicators may include compression of trachea, tracheal deviation, compression of other adjacent vital structures. Imaging often helps in correlating the symptoms with size of goiter, presence of tracheal deviation or extent of retrosternal extension. The cause for acute airway obstruction/orthopnea needs to be considered which may be due to haemorrhage within the thyroid gland or may be secondary to prolonged mechanical pressure with acute laryngeal edema and congestion. Although rare these acute problems can contribute for around 5-11\% and may lead to catastrophic consequences providing a clear indication for thyroidectomy in patients with retrosternal goiter. ${ }^{8}$ The majority of nodular goitres, limited to the neck and including a retrosternal component are often benign in nature. Clinical and ultrasonography examination can be done for cervical goitres and needle biopsy of suspicious areas can be taken, with cytological determination of malignant nodules, leading to patient selection for surgery. Retrosternal or subternal components of goitres are not easily imaged by ultrasound due to arte fact generated by bony structures and needle biopsies are difficult to perform in routine practice. This can lead to exclusion of malignancy thought with difficulty in retrosternal goitres. Prospective studies reveals the incidence of development of malignancy in goitres is $1.3-3.7$ new cases per 1000 patients. ${ }^{9,10} \mathrm{~A}$ recent review of evidence-based management of substernal goitres concluded the incidence of malignant transformation is equivalent in retrosternal goitres to those residing entirely in the neck. ${ }^{10}$

With few exceptions huge goiters can be removed by cervical approach but less than $2 \%$ patients require conventional cervical approach combined with sternotomy/ manubriotomy/ thoracotomy. ${ }^{11}$ Review of the literature regarding the complications of recurrent laryngeal nerve injury, hyperthyroidism, hyperparathyroidism and tracheomalacia after retrosternal goitre excision reveals conflicting results as to whether these specific complications are increased by comparison to cases of excision of simple cervical goitre. ${ }^{8,11,12}$ Total Thyroidectomy for retrosternal goitres with associated medical comorbidities should be performed by surgeons who are experienced in thyroid surgery to prevent complications and a cervical approach is successful in the maximum number of cases. Graves's disease is one of the most common cause for thyrotoxicosis or thyroid storm. It can be precipitated by systemic insults like trauma, surgery, trauma, myocardial infarction, pulmonary thromboembolism and severe infection. In the past, thyroid storm was most commonly caused in thyroid surgery patients who had uncontrolled hyperthyroidism. Though this condition has been decreased by newer imaging modalities, but has not been completely eliminated the incidence of thyroid storm. ${ }^{13}$ 
Preoperative management of the thyrotoxic patient includes preparation for elective or non urgent procedures and preparation for emergent procedures. When the case is non emergent, there must be control of thyrotoxicosis to achieve euthyroidism before surgery. In this cases, thionamide therapy preferably Neomercazole would be recommended and would facilitate euthyroidism within several weeks. ${ }^{14,15}$ The use of iodine (lugols iodine) as a method of decreasing thyroid vascularity and friability before thyroid surgery. Postoperatively Beta adrenergic receptors may be required for a period of 1 week. In our patient, Tab Neomarkazole $2.5 \mathrm{mg}$, Tab Inderal $80 \mathrm{mg}$ and Lugol Iodine solution orally were given for 1 week preoperatively to avoid Thyrotoxicosis followed by 5days postoperatively. ${ }^{16,17}$ There is always a controversy to perform a Total thyroidectomy in patients with retrosternal goitre. There was historical surgical dogma to operate the patient with retrosternal goitre. But due to increased use of radiological investigations, particularly advanced Computed tomography scans has identified the often asymptomatic retrosternal goitre. These developments have prompted some thyroid surgeons to challenge the traditional surgical doctrine. Today it remains generally accepted that patients of retrosternal goitre with clinical symptoms of stridors/dysphagia and/or radiological evidence of significant tracheal narrowing, esophageal compression or SVC syndrome.

\section{Conclusion}

Total Thyroidectomy in retorsternal goitre with hyperthyroidism patients must be performed with utmost precautions. Thyrotoxicosis and thyroid storm pose a critical diagnostic and therapeutic challenge to the clinician. Recognition of life-threatening thyrotoxicosis and prompt use of the arsenal of medications aimed to prevent the thyrotoxic process at every level is essential to successful management. Surgery is usually associated with increased risk of complications and hence must be performed by an experienced surgeon.

\section{Acknowledgements}

None.

\section{Conflict of interest}

Author declares that there is no conflict of interest.

\section{References}

1. Huins CT, Georgalas C, Mehrzad H, et al. A new classification system for retrosternal goitre based on a systematic review of its complications and management. International journal of surgery. 2008;6(1):71-76.

2. Chauhan, Ajay, and Jonathan W. Serpell. Thyroidectomy is safe and effective for retrosternal goitre. ANZ journal of surgery. 2006;76(4):238242 .
3. Rugiu MG, Piemonte M. Surgical approach to retrosternal goitre: do we still need sternotomy? Acta otorhinolaryngologica Ital. 2009;29(6):331338.

4. Hashmi SM, Premachandra DJ, Bennett AM, et al. Management of retrosternal goitres: results of early surgical intervention to prevent airway morbidity, and a review of the English literature. The Journal of Laryngology \& Otology. 2006;120(8):644-649.

5. McKay GD, Ng TH, Morgan GJ, et al. Giant functioning parathyroid cyst presenting as a retrosternal goitre. ANZ journal of surgery. 2007;77(4):297-304.

6. Shah PJ, Bright T, Singh SS, et al. Large retrosternal goitre: a diagnostic and management dilemma. Heart, Lung and Circulation. 2006;15(2):151152.

7. Wheeler MH. Retrosternal goitre. British journal of surgery. 1999;86(10):1235-1236.

8. Bennett AM, Hashmi SM, Premachandra DJ, et al. The myth of tracheomalacia and difficult intubation in cases of retrosternal goitre. The Journal of Laryngology \& Otology. 2004;118(10):778-780.

9. Riffat F, Del Pero MM, Fish B, et al. Radiologically predicting when a sternotomy may be required in the management of retrosternal goiters. Annals of Otology, Rhinology \& Laryngology. 2013;122(1):15-19.

10. Tsang, Flora HF, Lee TW, et al. Management of retrosternal goitre with superior vena cava obstruction. Heart, Lung and Circulation. 2007;16(4):312-314.

11. Armour RH. Retrosternal goitre. British Journal of Surgery. 2000;87(4):519-519.

12. Cooper, John C, Richard Nakielny, et al. The use of computed tomography in the evaluation of large multinodular goitres. Annals of the Royal College of Surgeons of England. 1991;73(1):32.

13. Madjar, Shahar, Dov Weissberg. Retrosternal goiter. Chest.1995;108(1):78-82.

14. Qureishi A, Garas G, Tolley N, et al. Can pre-operative computed tomography predict the need for a thoracic approach for removal of retrosternal goitre? International journal of surgery. 2013;11(3):203-208.

15. Abraham D, Singh N, Lang B, et al. Benign nodular goitre presenting as acute airway obstruction. ANZ journal of surgery. 2007;77(5):364-367.

16. Dave ST, Kamath SK, Shetty AN, et al. Anaesthesia management for subtotal thyroidectomy in a case of multinodular goitre with retrosternal extension and superior vena caval syndrome. Journal of postgraduate medicine. 2001;47(3):219.

17. Xu J, Shen B, Li Y, et al. Enormous goiter in posterior mediastinum: report of 2 cases and literature review. Journal of the Formosan Medical Association. 2009;108(4):337-343. 